\title{
The prognostic value of prognostic nutritional index (PNI) and neutrophil to lymphocyte ratio (NLR) for advanced non-small cell lung cancer treated with platinum-based chemotherapeutics
}

\author{
Jian Wang ${ }^{1,2 \#}$, Yiqian Liu ${ }^{1 \#}$, Xiaoguang $\mathrm{Mi}^{3}$, Mengting Shao ${ }^{2}$, Lingxiang Liu ${ }^{1}$ \\ ${ }^{1}$ Department of Oncology, Cancer Rehabilitation Center, The First Affiliated Hospital of Nanjing Medical University, Nanjing 210029, China; \\ ${ }^{2}$ Department of Oncology, ${ }^{3}$ Department of Respiratory Medicine, The Fifth People's Hospital of Changshu, Changshu 215500, China \\ Contributions: (I) Conception and design: J Wang, Y Liu, L Liu; (II) Administrative support: L Liu; (III) Provision of study materials or patients: \\ J Wang, Y Liu, L Liu; (IV) Collection and assembly of data: X Mi, M Shao; (V) Data analysis and interpretation: J Wang, Y Liu; (VI) Manuscript \\ writing: All authors; (VII) Final approval of manuscript: All authors. \\ \#These authors contributed equally to this work. \\ Correspondence to: Lingxiang Liu. Department of Oncology, Cancer Rehabilitation Center, The First Affiliated Hospital of Nanjing Medical \\ University, Nanjing 210029, China. Email: 1lxlau@163.com.
}

Background: The present study aimed to investigate the relationship between prognostic nutritional index (PNI) and peripheral blood neutrophil to lymphocyte ratio (NLR), and the prognosis of patients with advanced non-small cell lung cancer (NSCLC) treated with platinum-based therapeutics.

Methods: The data of 99 advanced NSCLC patients treated with platinum chemotherapeutics between January 2011 and June 2019 were retrospectively analyzed. The association between PNI and NLR and the clinicopathological characteristics of the patients was examined. The patients were randomized into high or low groups according to PNI and NLR. The predictive value of PNI and NLR for overall survival (OS) was evaluated by receiver operating characteristic (ROC) curve analysis. Univariate and multivariate Cox proportional hazards regression analyses were performed to investigate the prognostic factors of advanced NSCLC patients treated with platinum-based chemotherapeutics. The association between PNI and NLR and progression-free survival (PFS) or OS was determined using the Kaplan-Meier method and compared between groups using the log-rank test.

Results: The ROC curve analysis determined the optimal cut-off values of PNI and NLR for predicting OS to be 52.525 and 3.525 , respectively. Univariate analysis indicated that low Karnofsky performance scale (KPS) score $(\mathrm{P}=0.005)$, poor tumor differentiation $(\mathrm{P}=0.022)$, brain metastasis $(\mathrm{P}<0.001)$, and low $\mathrm{PNI}$ $(\mathrm{P}=0.001)$ were independent risk factors for PFS in patients with advanced NSCLC; however, there was no significant correlation observed between NLR $(\mathrm{P}=0.082)$ and PFS in patients with advanced NSCLC. Low KPS score $(\mathrm{P}=0.003)$, poor tumor differentiation $(\mathrm{P}=0.001)$, brain metastasis $(\mathrm{P}<0.001)$, low $\mathrm{PNI}(\mathrm{P}<0.001)$, and high NLR ( $\mathrm{P}=0.046)$ were significantly associated with shorter OS. Furthermore, Cox multivariate analysis revealed that brain metastasis $(\mathrm{P}=0.005)$ and low PNI $(\mathrm{P}=0.008)$ were significant independent prognostic factors for PFS, while brain metastasis $(\mathrm{P}=0.003)$ and low $\mathrm{PNI}(\mathrm{P}=0.028)$ were also found to be significant independent risk factors for poor OS.

Conclusions: PNI is a reliable, simple, easily available, and inexpensive biomarker for predicting the prognosis of advanced NSCLC patients treated with platinum-based chemotherapeutics in routine clinical practice. Furthermore, PNI is superior to NLR in as a prognostic indicator for advanced NSCLC patients treated with platinum-based chemotherapeutics.

Keywords: Prognostic nutritional index (PNI); neutrophil to lymphocyte ratio (NLR); lung neoplasms; efficacy of chemotherapy; overall survival time (OS time) 
Submitted Feb 20, 2020. Accepted for publication Apr 07, 2020.

doi: 10.21037/apm.2020.04.31

View this article at: http://dx.doi.org/10.21037/apm.2020.04.31

\section{Introduction}

Lung cancer continues to be the most frequently diagnosed malignant tumor in the world, and is the leading cause of cancer-associated mortality (1). The predominant histologic subtype of lung cancer, non-small cell lung cancer (NSCLC), constitutes approximately 80-85\% of all lung cancer cases. Currently, surgical resection is established as the gold standard in first-line treatment for early-stage NSCLC patients. However, in the majority of cases, NSCLC is initially diagnosed at the advanced stage, at which point surgical resection alone cannot provide a complete cure. For patients with advanced NSCLC, platinum-based chemotherapy remains the standard of care $(2,3)$. Overall, the prognosis for these patients is poor, and little improvement has been observed in survival over the past few decades.

NSCLC treatment decisions are typically based on the patient's health status and the crucial prognostic factors include stage, histological subtype, cell differentiation, and the molecular characteristics of NSCLC. Late TNM stage, poor tumor differentiation and no mutation of EGFR are generally recognized as the poor prognostic factors of NSCLC. However, at present, patients who are receiving the same treatment strategy cannot be significantly stratified according to these and other clinicopathological characteristics. Therefore, the identification of promising, accessible and reliable prognostic predictive factors by which to stratify patients is of great significance.

An increasing number of studies have reported that immunological status, comprising inflammatory status and nutritional status, remains a significant predictive factor for the prognosis of patients with malignant tumors. Prognostic nutritional index (PNI), a simple index which is calculated by combining the serum albumin concentration and total peripheral blood lymphocyte count, has been increasingly applied to assess the immune-nutritional status of patients who undergo surgery. Multiple studies have suggested that PNI can facilitate effective evaluation of the prognosis of patients with various cancers, including stomach (4), colorectal (5), breast (6), and cervical cancer (7). Moreover, locoregional tumor cell growth, tumor cell metastasis, and tumor immunity suppression are promoted by systemic inflammatory response (8), in which neutrophils and lymphocytes serve as important components. Neutrophil to lymphocyte ratio (NLR), an index of inflammation, has been extensively studied for its role in cancer prognosis. However, there is a paucity of literature regarding the impact of PNI and NLR in the prognosis of advanced NSCLC patients following platinum-based chemotherapy. Therefore, the present study was initiated to investigate the clinical significance of PNI and NLR in predicting the prognosis of patients with advanced NSCLC who receive platinum-based chemotherapy.

\section{Methods}

\section{Study subjects}

This retrospective study analyzed patients with advanced NSCLC who received chemotherapeutic treatment in the Department of Oncology of the Fifth People's Hospital of Changshu, China in the period between January, 2011 and June, 2019.

Patients who met the following criteria were included: (I) histologically confirmed primary NSCLC; (II) had undergone complete blood laboratory and blood biochemical investigation in the week preceding the commencement of chemotherapy; (III) no previous history of tumor-related treatment; and (IV) had complete followup data. Patients who met any of the following criteria were excluded: (I) had experienced infection in the 2 weeks prior to surgery; (II) had received hormonal, non-steroidal anti-inflammatory, granulocyte colony-stimulating factor or other drug therapies in the month before treatment; (III) presented with comorbidities including coronary heart disease, rheumatoid disease, diabetes, and other diseases; (IV) patients with malignancy in other organs or hematological diseases; $(\mathrm{V})$ a follow-up time of less than 3 months or loss to follow-up; or (VI) patients with digestive tract diseases affecting the digestion and absorption of food.

\section{Data collection}

Clinicopathological characteristics data were retrieved 
from the patients' electronic medical records, including gender, age, histological type, tumor differentiation, chemotherapy regimen, Karnofsky performance scale (KPS) score, smoking history, brain metastasis, blood laboratory investigations, and biochemical indexes within the 1 week before treatment. Two $\mathrm{mL}$ of peripheral blood venous blood samples were collected by EDTA-K2 anticoagulant vacuum test tube, and the venous blood in the tube was quickly mixed with the anticoagulant. Mindray BC-6800 blood cell analyzer was used to select the automatic whole blood model to analyze the samples in the state of readiness for counting, and various indexes of complete blood count were obtained. Five $\mathrm{mL}$ of peripheral venous blood samples were collected by coagulant stimulating vacuum test tube, and the serum was centrifuged by a centrifuge. The biochemical indexes were determined by the Toshiba TBA120 automatic biochemical analyzer. The values of PNI and NLR were calculated based on the test indices. PNI was calculated by serum albumin value $(\mathrm{g} / \mathrm{L})+5 \times$ peripheral blood lymphocyte count $\left(\times 10^{9} / \mathrm{L}\right)$, and NLR was defined as the neutrophil count divided by the lymphocyte count.

\section{Follow-up}

All patients were administered systemic platinum-based chemotherapy. Each patient attended regular follow-ups until June 2019 or death. Follow-up examinations were performed at regular 3-month intervals in the first-year post-chemotherapy, 6-month intervals over the subsequent 2 years, and on an annual basis thereafter. After the treatment was completed, regular follow-up was performed, and the curative effect was evaluated according to the response evaluation in solid tumors (RECIST) evaluation criteria. Tumor response was classified as: complete remission (CR), partial remission (PR), disease stability (SD), or disease progression (PD). Progression-free survival (PFS) was defined as the period from the date of initial pathological diagnosis to the date of $\mathrm{PD}$, death because of any cause, or the date of last follow-up (months). Overall survival (OS) time was calculated as the period from the first date of pathological diagnosis to the date of death or last follow-up (months).

\section{Statistical analysis}

Continuous variables were expressed as mean \pm standard deviation, and differences between groups were compared through one-way analysis of variance. Categorical variables were expressed as number and percentage, with differences between groups determined using the $\chi^{2}$ test. The hematological index values of the patient study were considered as the variable and the short-term response of platinum-based chemotherapy as the end-point. The optimal cutoff values were determined by using the receiver operating characteristic (ROC) curve to predict OS and PFS. Survival curves were calculated by the Kaplan-Meier method, and the survival curves were compared using the $\log$-rank test. Multivariate analysis was carried out with Cox proportional hazards model, and all of the variables found to be significant in univariate analysis were entered into the multivariate analysis. All statistical analysis was performed using Statistical Package for the Social Sciences (SPSS, version 19.0; IBM Corp., Armonk, NY, USA).

\section{Results}

\section{Clinicopathological characteristics of patients with advanced NSCLC following platinum-based therapy}

Of 114 patients with advanced NSCLC who were initially enrolled in this study, 99 patients were ultimately included. The excluded patients included, 6 cases who had received antineoplastic therapy, 3 patients who had experienced infection in the 2 weeks before treatment, 5 patients who had received drugs that affected the results of blood laboratory examination 1 month before treatment, and 1 patient who had a tumor in another organ. The baseline clinicopathological characteristics of the eligible patients are summarized in Table 1 .

\section{Optimal cut-off values}

The optimal cutoff values for the prediction of OS were $52.525(\mathrm{P}=0.023)$ and $3.525(\mathrm{P}=0.036)$ for $\mathrm{PNI}$ and NLR, respectively (Figure 1).

\section{The association between PNI and NLR indices and the clinicopathological characteristics of patients with advanced NSCLC following platinum-based therapy}

Based on the optimal cut-off values of PNI and for NLR described above, the patients were separated into two groups of high or low levels for PNI and NLR, respectively. Subsequently, differences in age, sex, smoking status, KPS 
Table 1 Clinicopathological characteristics of patients with advanced NSCLC

\begin{tabular}{|c|c|}
\hline Characteristics & $\mathrm{N}(\%)$ \\
\hline \multicolumn{2}{|l|}{ Age (years) } \\
\hline$<60$ & $25(25.3)$ \\
\hline$\geq 60$ & $74(74.7)$ \\
\hline \multicolumn{2}{|l|}{ Gender } \\
\hline Male & $66(66.7)$ \\
\hline Female & 33 (33.3) \\
\hline \multicolumn{2}{|l|}{ Smoking history } \\
\hline Smoker & $53(53.5)$ \\
\hline Non-smoker & $46(46.5)$ \\
\hline \multicolumn{2}{|l|}{ KPS score } \\
\hline$\geq 70$ & $77(77.8)$ \\
\hline$<70$ & $22(22.2)$ \\
\hline \multicolumn{2}{|l|}{ Histological type } \\
\hline Squamous & $45(45.5)$ \\
\hline Adenocarcinoma & $54(54.5)$ \\
\hline \multicolumn{2}{|l|}{ Tumor differentiation } \\
\hline Poorly differentiated & $43(43.4)$ \\
\hline Moderately-well differentiated & $56(56.6)$ \\
\hline \multicolumn{2}{|l|}{ Brain metastasis } \\
\hline Without brain metastasis & $91(91.9)$ \\
\hline With brain metastasis & $8(8.1)$ \\
\hline \multicolumn{2}{|l|}{ Chemotherapy regimen } \\
\hline With cisplatin & $51(51.5)$ \\
\hline With carboplatin & $48(48.5)$ \\
\hline \multicolumn{2}{|l|}{ PNI } \\
\hline$\geq 52.525$ & $45(45.5)$ \\
\hline$<52.525$ & $54(54.5)$ \\
\hline \multicolumn{2}{|l|}{ NLR } \\
\hline$<3.525$ & $59(59.6)$ \\
\hline$\geq 3.525$ & $40(40.4)$ \\
\hline $\begin{array}{l}\text { NSCLC, non-small cell lu } \\
\text { performance scale; PNI, pros } \\
\text { neutrophil to lymphocyte ratio. }\end{array}$ & $\begin{array}{l}\text { Karnofs } \\
\text { index; N }\end{array}$ \\
\hline
\end{tabular}

score, tumor type, tumor differentiation, brain metastasis, and chemotherapy regimen were compared between the high and low groups for PNI and NLR. The results indicated that there were no significant correlations between PNI, NLR, and the above-mentioned clinicopathological characteristics (Table 2).

The association between PNI and NLR indices and the prognosis of patients with advanced NSCLC following platinum-based therapy

Univariate and multivariate survival analyses of PFS in patients with advanced NSCLC

Univariate analysis revealed low KPS score, poor tumor differentiation, brain metastasis, and low PNI to be significant prognostic factors for reduced PFS in patients with advanced NSCLC; however, there was no significant correlation between NLR and PFS in patients with advanced NSCLC (Figure 2). Multivariate analysis indicated that brain metastasis and low PNI were significant independent prognostic factors of poor PFS $(\mathrm{P}=0.005$ and $\mathrm{P}=0.008$, respectively; Table 3).

\section{Univariate and multivariate survival analysis of $O S$ in} patients with advanced NSCLC

Univariate analysis revealed that low KPS score, poor tumor differentiation, brain metastasis, low PNI, and high NLR were significantly correlated with reduced OS (Figure 3). Further multivariate analysis indicated that brain metastasis and low PNI were significant prognostic factors of poor OS (Table 4).

\section{Discussion}

Platinum-based chemotherapy has taken up its places a first-line therapy for advanced NSCLC; however, the development of chemotherapy resistance is inevitable in patients with the disease, which presents a significant obstacle in effective tumor management $(9,10)$. Clinical decision-making for the treatment of advanced NSCLC patients is dependent on appropriate prognostic indicators, which can guide clinicians in predicting the efficacy of anti-tumor therapy. Therefore, this study investigated 

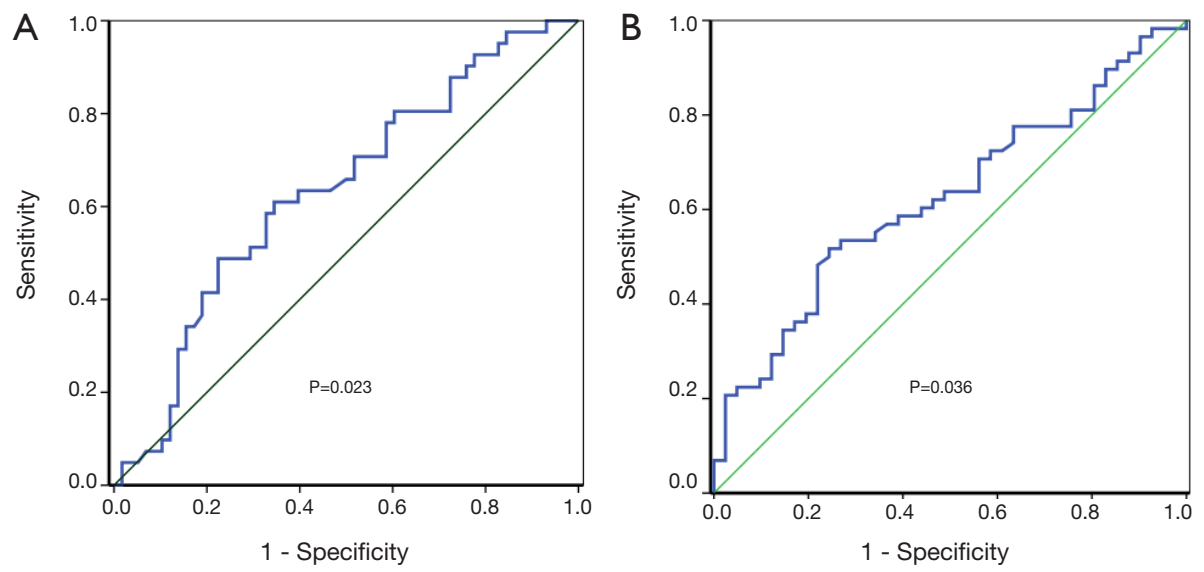

Figure 1 The ROC analysis of PNI and NLR to predict the prognosis of advanced NSCLC patients treated with platinum-based chemotherapeutics. (A) The ROC curve analysis of pre-treatment PNI in predicting the prognosis of advanced NSCLC patients (0.023); (B) the ROC curve analysis of pre-treatment NLR in predicting the prognosis of advanced NSCLC patients (0.036). ROC, receiver operating characteristic; PNI, prognostic nutritional index; NLR, neutrophil to lymphocyte ratio; NSCLC, non-small cell lung cancer.

the prognostic significance of NLR and PNI as markers of systemic inflammation in patients with advanced NSCLC who received platinum-based chemotherapy. The results indicated that NLR value had a significant effect on the prognosis of these patients. Moreover, PNI and brain metastasis were also found to serve as independent prognostic factors for advanced NSCLC patients treated with platinum-based chemotherapy.

Immunological status, comprising nutritional status and inflammatory status, is of great significance to the survival of patients with various cancer types, including NSCLC. PNI and NLR reflect the patient's immunonutritional status and inflammation status, respectively. Both the PNI and NLR indices are accurate, inexpensive, highly reproducible, and widely used blood-based investigations.

PNI, which was originally proposed by Smale et al. (11), predominantly evaluates the risk of recurrence and survival following surgical treatment; however, owing to the complex calculation it entails, it was not taken up explicitly at that time. In 1984, Onodera et al. simplified the formula for the calculation of PNI, which was calculated based on the two indices of serum albumin and lymphocyte count (12). PNI was once used to evaluate the nutritional and immune status of gastrointestinal surgery patients. In recent years, an increasing number of studies have advocated the use of PNI evaluation for the prognosis of patients with malignant tumors, including lung cancer; moreover, the ability of PNI to predict the outcome of patients with tumors, regardless of the location and primary site of origin of the tumor, has also been presented (4,13-16).

Alterations in metabolic and nutritional rate, including malnutrition and metabolic rate, can significantly influence the survival and recovery of cancer patients. Notably, serum albumin deficiency represents both poor nutritional status and poor prognosis $(17,18)$. As important immune cells, lymphocytes play a crucial role in immune surveillance through their inhibition of the proliferation, invasion, and migration of tumor cells $(19,20)$. Furthermore, malnutrition and an impaired immune system can collectively influence tolerance and response to chemotherapeutic treatments, which can potentially lead to reduced survival in cancer patients $(21,22)$.

Because relatively few studies have examined PNI in patients with NSCLC, the optimal cut-off value for PNI remains undefined. Due to heterogeneity among patents and low sample sizes, the cut-off values vary among different studies. In our study, the optimal cut-off value of PNI, which was calculated based on the ROC curve, was 52.525. The OS of NSCLC patients following platinumbased chemotherapy with PNI $\geq 52.525$ was observed to be significantly higher than that of patients with $\mathrm{PNI}<52.525$. The optimal cut-off value of PNI indicated by Shimizu et al. (23) was 50, which was lower than our 52.525, while the value reported by Jin et al. (24) was 53.85, which was higher than our PNI value. Moreover, based on multivariate analysis, PNI was found to be an independent prognostic factor in the present study. This finding is consistent with the results of Dai et al. (25) Furthermore, PNI has also been 
Table 2 The association between clinicopathological characteristics of advanced NSCLC patients and high and low indices for PNI and NLR

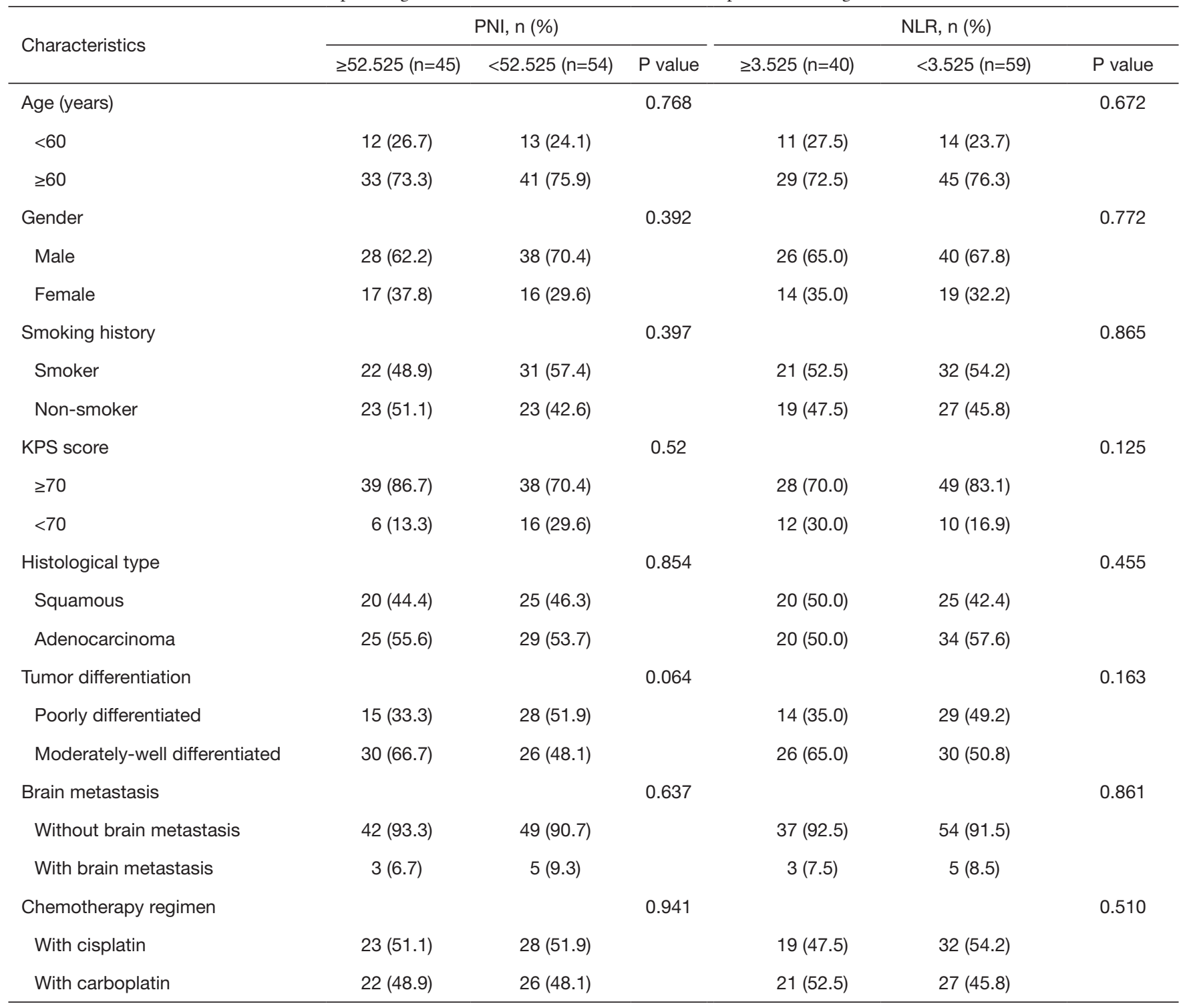

NSCLC, non-small cell lung cancer; PNI, prognostic nutritional index; NLR, neutrophil to lymphocyte ratio; KPS, Karnofsky performance scale.

reported to be significantly correlated with gender and histology (26). The incidence of low PNI status in female patients with lung cancer has been revealed to be lower than in male patients. Similarly, low PNI has been found to be rarer in patients with adenocarcinoma than in those with non-adenocarcinoma. However, this is in contrast with the results of the present study. Considering the limited sample size of the present study, our results warrant further validation with a large sample size.

NLR, comprising neutrophil technique and lymphocyte count, serves as an effective biomarker for detecting the inflammatory state of the immune system (27). Neutrophils can contribute to the proliferation and survival of malignant cells and promote metastasis and angiogenesis by producing proangiogenic chemokines and growth factors $(27,28)$. In contrast, lymphocytes produce cytokines, which play an important role in tumor defense and immune surveillance through the induction of cytotoxic cell death and inhibition of tumor cell proliferation and migration (29). NLR can reflect a balance between the protumor and antitumor 

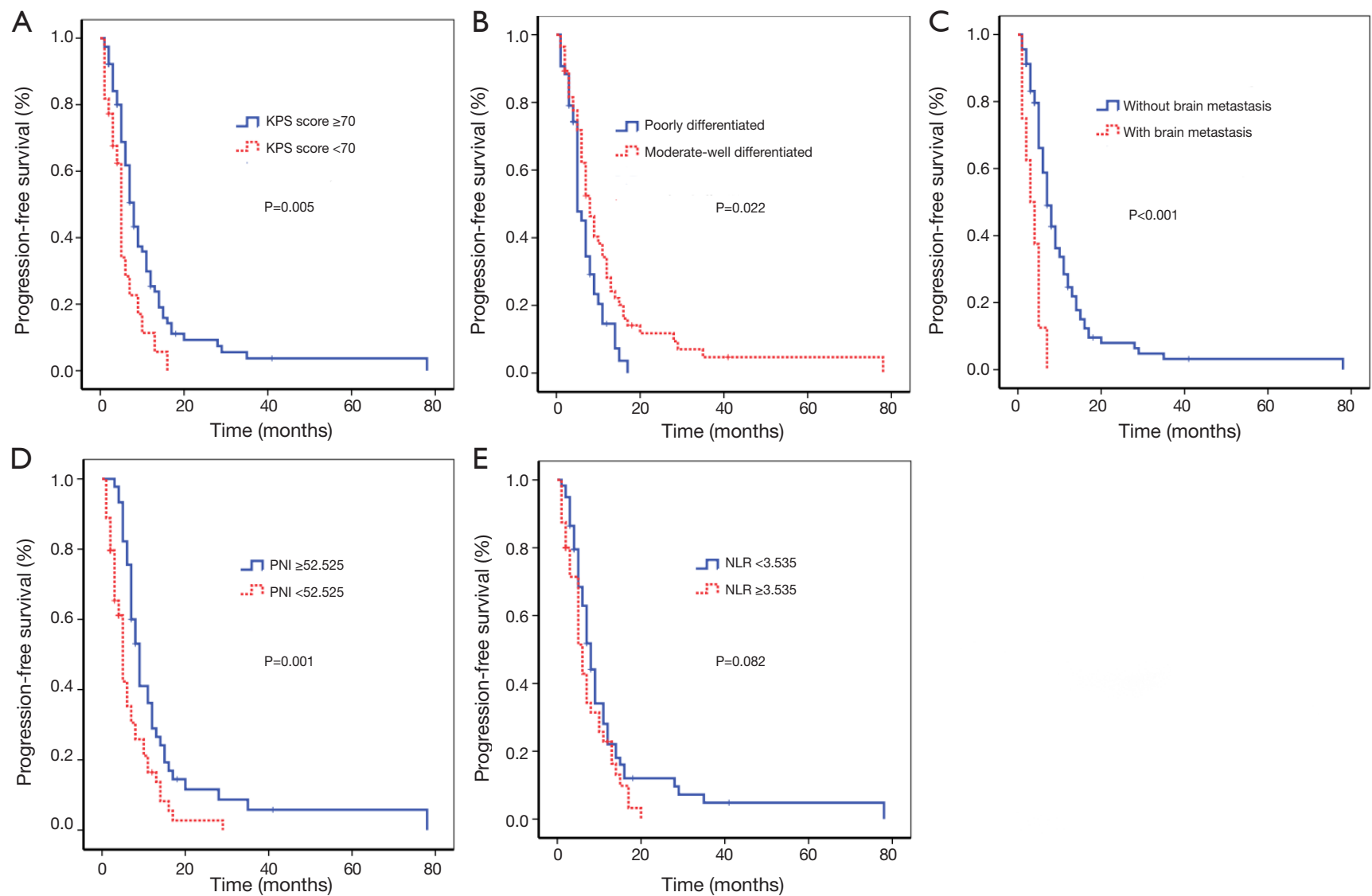

Figure 2 The association between the patients' clinicopathological characteristics and PFS. (A) The effect of KPS score on PFS (P=0.005); (B) the effect of differentiation degree on PFS ( $\mathrm{P}=0.022)$; (C) the effect of brain metastasis on PFS (P<0.001); (D) the effect of PNI on PFS $(\mathrm{P}=0.001)$; (E) the effect of NLR on PFS ( $\mathrm{P}=0.082)$. PFS, progression-free survival; KPS, Karnofsky performance scale; PNI, prognostic nutrition index; NLR, neutrophil to lymphocyte ratio.

inflammatory status of patients with cancer; thus, any alteration in neutrophil and lymphocyte counts can be associated with cancer progression (30,31).

Although univariate analysis of this study revealed NLR to be a significant prognostic factor for OS of patients with advanced NSCLC, multivariate regression analysis suggested that NLR is not an independent prognostic factor for OS in advanced NSCLC patients treated with platinumbased chemotherapy. Furthermore, this study also indicated that PNI is superior to NLR for the prognosis of patients with advanced NSCLC lung cancer who receive platinum chemotherapy. However, these findings are in contrast to the results of many previous studies (32-34). The differences in the findings might be attributed to the following: (I) variability in the number of cases, stage, and driver gene mutation status among the study subjects: All of the 99 patients in our study had stage IVNSCLC. However, the number of cases in the three studies mentioned varied from 109 to 1,225 . In addition to patients with stage IV, these studies also included some patients with stage III disease. Besides this, we were unable to collect the complete data on patient-driver gene status due to the limited availability of data. A previous study by Zhang et al. included patients with EGFR mutations (33). In addition to patients with EGFR mutations, there were different proportions of EGFR-negative or patients with unknown EGFR-mutation status in the two studies $(32,34)$. (II) The treatment scheme of the patients was different among different studies: we administered platinum-based chemotherapy. Zhang et al. treated patients with EGFR-TKI therapy, while the subjects in the other two studies $(32,34)$ were prescribed nivolumab immunotherapy. (III) The time the peripheral 
Table 3 Univariate and multivariate analyses of progression-free survival in the advanced NSCLC patients

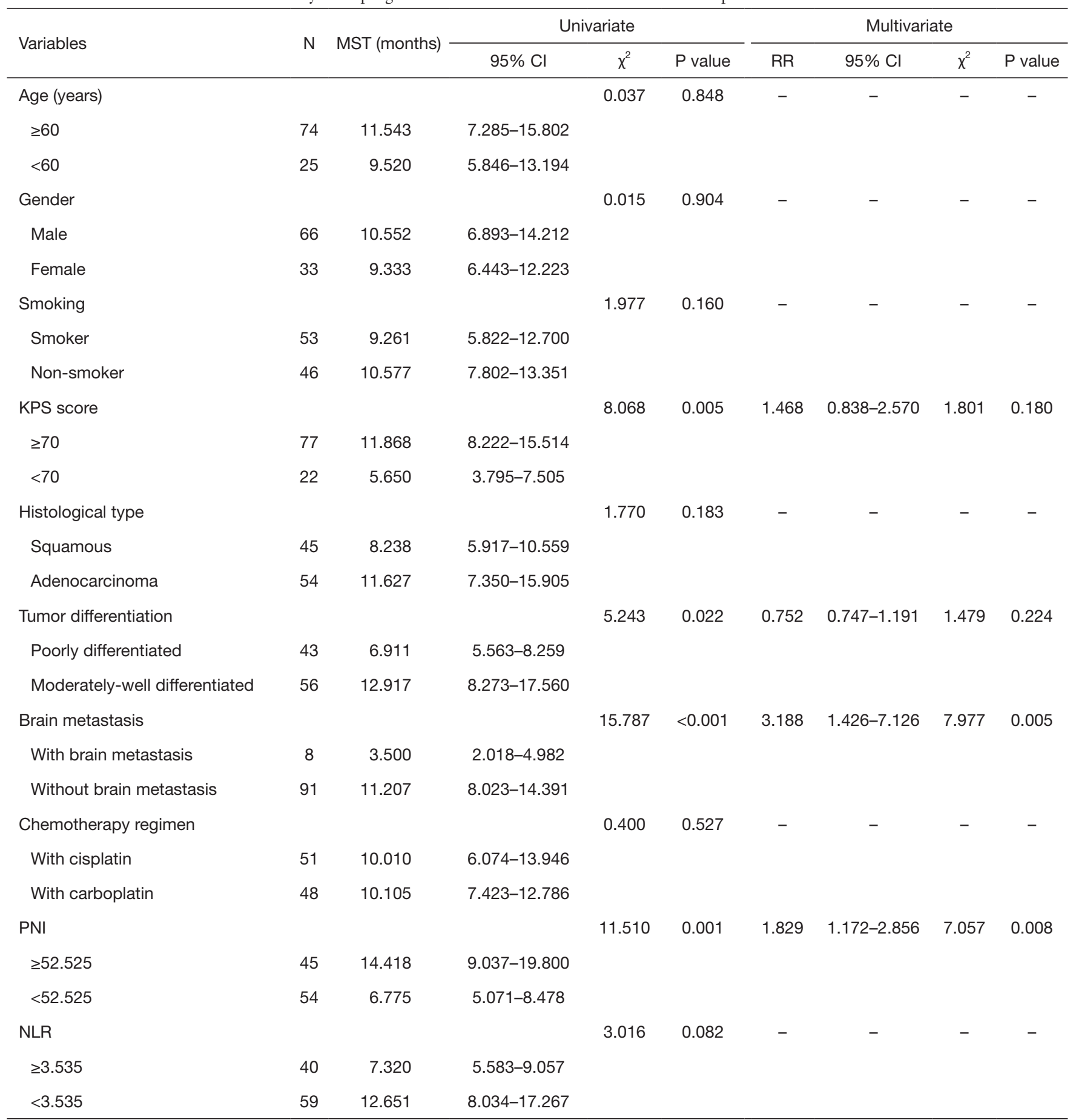

NSCLC, non-small cell lung cancer; MST, mean survival time; KPS, Karnofsky performance scale; PNI, prognostic nutrition index; NLR, neutrophil to lymphocyte ratio; $\mathrm{Cl}$, confidence interval; $\mathrm{RR}$, relative risk. 

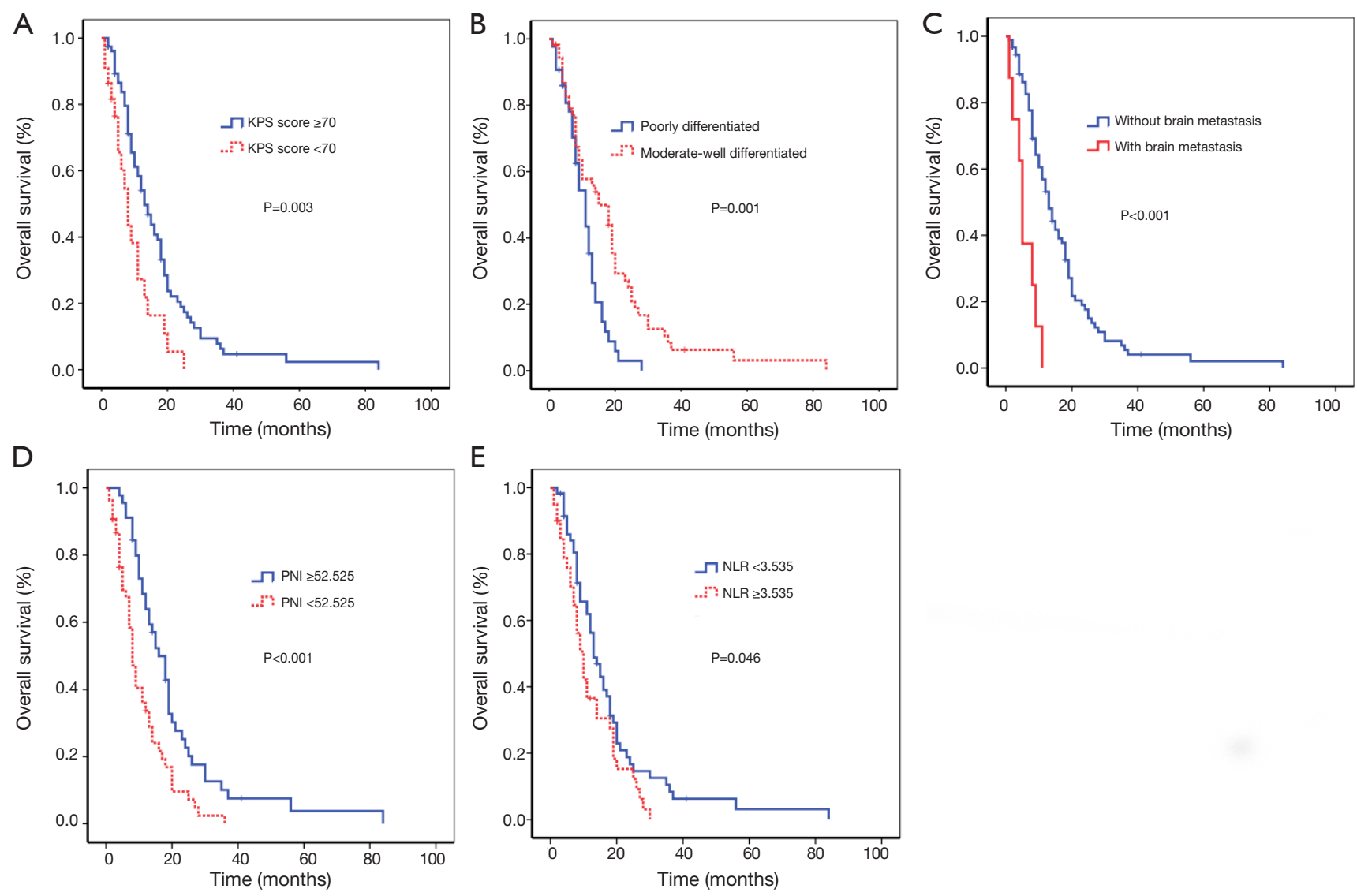

Figure 3 The association between clinicopathological characteristics and OS. (A) The effect of KPS score on OS (P=0.003); (B) the effect of differentiation degree on OS $(\mathrm{P}=0.001)$; (C) the effect of brain metastasis on OS $(\mathrm{P}<0.001)$; (D)the effect of $\mathrm{PNI}$ on $\mathrm{OS}(\mathrm{P}<0.001)$; (E) the effect of NLR on OS (P=0.046). OS, overall survival; KPS, Karnofsky performance scale; PNI, prognostic nutritional index; NLR, neutrophil to lymphocyte ratio.

blood samples were collected varied considerably among the different studies: in Zhang et al.'s study, peripheral blood samples were collected within the week preceding treatment, and Cao et al. (34) and Khunger et al. (32) implied that there was a significant correlation between NLR and OS after treatment.

However, to precisely determine the effects of immunonutrition status and inflammatory state on tumor prognosis and therapeutic efficacy, further large-scale prospective clinical studies are needed to address the following concerns: (I) determining a unified best cutoff value; (II) the influence of the dynamic changes of the indicators on the curative effect; (III) determining a unified application of a combination of conventional serum biomarkers for accurately predicting treatment response; (IV) evaluating of the effects of intervening predictive indicators for improving the survival of patients.

This study has several limitations. Firstly, this was a retrospective, single-center study with small sample size. Secondly, PNI and NLR data were collected within the 1 -week preceding treatment, and fluctuations in PNI and NLR throughout the entire treatment procedure and follow-up were not fully recorded and analyzed. This is noteworthy because these data may change according to time and conditions during treatment. Thirdly, many other biologic markers were not included in the evaluation due to the lack of data on the side effects of chemotherapy. Therefore, further large, multicenter studies are warranted to explicitly determine the effectiveness and accuracy of PNI and NLR for predicting the prognosis of advanced NSCLC patients treated with platinum-based chemotherapy. The heterogeneity of the subjects included in our study is high, 
Table 4 Univariate and multivariate analyses of overall survival in the advanced NSCLC patients

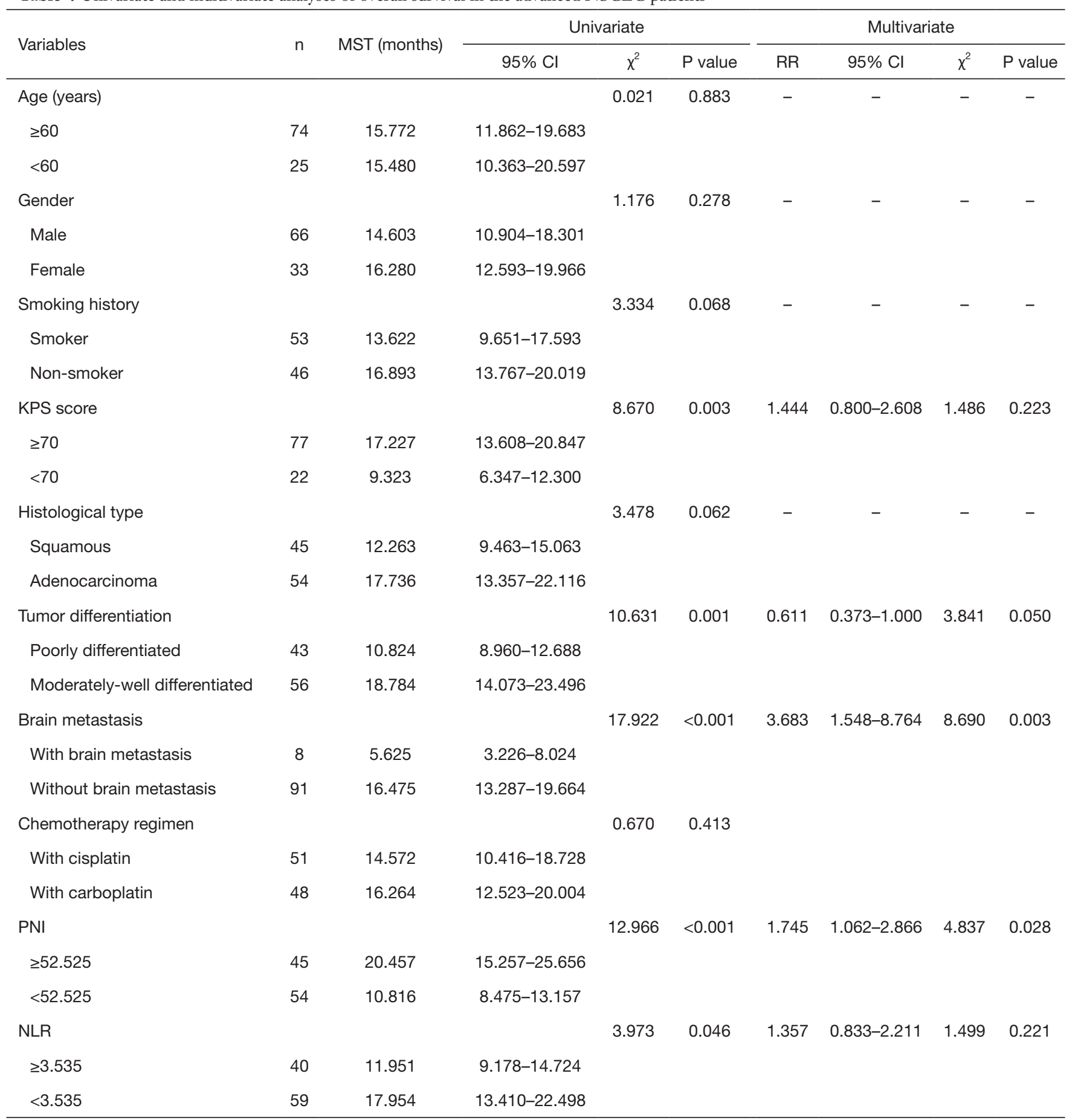

NSCLC, non-small cell lung cancer; MST, mean survival time; KPS, Karnofsky performance scale; PNI, prognostic nutrition index; NLR, neutrophil to lymphocyte ratio; $\mathrm{Cl}$, confidence interval; $\mathrm{RR}$, relative risk. 
so one of the subgroups, such as patients with EGFR mutation, can be selected for analysis in the next study. Prospective studies could also be designed to assess more aggressive treatment of patients with a perceived poor prognosis.

\section{Conclusions}

PNI is a reliable, simple, easily available, and inexpensive biomarker for predicting the prognosis of advanced NSCLC patients treated with platinum-based chemotherapeutics in routine clinical practice. Furthermore, a high PNI value may serve as an independent predictor of long-term OS. PNI is a superior prognostic indicator to NLR for determining the prognosis of advanced NSCLC patients treated with platinum-based chemotherapeutics.

\section{Acknowledgments}

Funding: This work was supported by the National Natural Science Foundation of China (No. 81472782) and Natural Science Foundation of Jiangsu Province of China (No. BK20141491).

\section{Footnote}

Conflicts of Interest: All authors have completed the ICMJE uniform disclosure form (available at http://dx.doi. org/10.21037/apm.2020.04.31). The authors have no conflicts of interest to declare.

Ethical Statement: The authors are accountable for all aspects of the work in ensuring that questions related to the accuracy or integrity of any part of the work are appropriately investigated and resolved. The study was approved by the Research Ethics Committee, The Fifth People's Hospital of Changshu (No. 2019008). Informed consent was exempted due to the retrospective nature of the study.

Open Access Statement: This is an Open Access article distributed in accordance with the Creative Commons Attribution-NonCommercial-NoDerivs 4.0 International License (CC BY-NC-ND 4.0), which permits the noncommercial replication and distribution of the article with the strict proviso that no changes or edits are made and the original work is properly cited (including links to both the formal publication through the relevant DOI and the license). See: https://creativecommons.org/licenses/by-nc-nd/4.0/.

\section{References}

1. Gorini G, Carreras G. Issues in implementing lung cancer screening in United States and Europe. Ann Transl Med 2018;6:S54.

2. Al-Saleh K, Quinton C, Ellis PM. Role of pemetrexed in advanced non-small-cell lung cancer: meta-analysis of randomized controlled trials, with histology subgroup analysis. Curr Oncol 2012;19:e9-15.

3. Lin CC. Challenges of the phase I drug development in nonsmall cell lung cancer. Chin Clin Oncol 2019;8:25.

4. Yang $\mathrm{Y}$, Gao $\mathrm{P}$, Song $\mathrm{Y}$, et al. The prognostic nutritional index is a predictive indicator of prognosis and postoperative complications in gastric cancer: A metaanalysis. Eur J Surg Oncol 2016;42:1176-82.

5. Tokunaga R, Sakamoto Y, Nakagawa S, et al. Prognostic Nutritional Index Predicts Severe Complications, Recurrence, and Poor Prognosis in Patients With Colorectal Cancer Undergoing Primary Tumor Resection. Dis Colon Rectum 2015;58:1048-57.

6. Mohri T, Mohri Y, Shigemori T, et al. Impact of prognostic nutritional index on long-term outcomes in patients with breast cancer. World J Surg Oncol 2016;14:170.

7. Haraga J, Nakamura K, Omichi C, et al. Pretreatment prognostic nutritional index is a significant predictor of prognosis in patients with cervical cancer treated with concurrent chemoradiotherapy. Mol Clin Oncol 2016;5:567-74.

8. Ferlay J, Soerjomataram I, Dikshit R, et al. Cancer incidence and mortality worldwide: sources, methods and major patterns in GLOBOCAN 2012. Int J Cancer 2015;136:E359-86.

9. Liu J, Wan L, Lu K, et al. The Long Noncoding RNA MEG3 Contributes to Cisplatin Resistance of Human Lung Adenocarcinoma. PLoS One 2015;10:e0114586.

10. Warner A, Dahele M, Hu B, et al. Factors Associated With Early Mortality in Patients Treated With Concurrent Chemoradiation Therapy for Locally Advanced NonSmall Cell Lung Cancer. Int J Radiat Oncol Biol Phys 2016;94:612-20.

11. Smale BF, Mullen JL, Buzby GP, et al. The efficacy of nutritional assessment and support in cancer surgery. Cancer 1981;47:2375-81.

12. Onodera T, Goseki N, Kosaki G. Prognostic nutritional index in gastrointestinal surgery of malnourished cancer patients. Nihon Geka Gakkai Zasshi 1984;85:1001-5.

13. Qiu C, Qu X, Shen H, et al. Evaluation of Prognostic 
Nutritional Index in Patients Undergoing Radical Surgery with Nonsmall Cell Lung Cancer. Nutr Cancer 2015;67:741-7.

14. Nakatani M, Migita K, Matsumoto S, et al. Prognostic significance of the prognostic nutritional index in esophageal cancer patients undergoing neoadjuvant chemotherapy. Dis Esophagus 2017;30:1-7.

15. Kang M, Chang CT, Sung HH, et al. Prognostic Significance of Pre- to Postoperative Dynamics of the Prognostic Nutritional Index for Patients with Renal Cell Carcinoma Who Underwent Radical Nephrectomy. Ann Surg Oncol 2017;24:4067-75.

16. Yang Y, Gao P, Chen X, et al. Prognostic significance of preoperative prognostic nutritional index in colorectal cancer: results from a retrospective cohort study and a meta-analysis. Oncotarget 2016;7:58543-52.

17. Laviano A, Di Lazzaro L, Koverech A. Nutrition support and clinical outcome in advanced cancer patients. Proc Nutr Soc 2018;77:388-93.

18. Kühn T, Sookthai D, Graf ME, et al. Albumin, bilirubin, uric acid and cancer risk: results from a prospective population-based study. Br J Cancer 2017;117:1572-9.

19. Shoji F, Morodomi Y, Akamine T, et al. Predictive impact for postoperative recurrence using the preoperative prognostic nutritional index in pathological stage I nonsmall cell lung cancer. Lung Cancer 2016;98:15-21.

20. Dunn GP, Bruce AT, Ikeda H, et al. Cancer immunoediting: from immunosurveillance to tumor escape. Nat Immunol 2002;3:991-8.

21. Miao J, Xiao W, Wang L, et al. The value of the Prognostic Nutritional Index (PNI) in predicting outcomes and guiding the treatment strategy of nasopharyngeal carcinoma (NPC) patients receiving intensity-modulated radiotherapy (IMRT) with or without chemotherapy. J Cancer Res Clin Oncol 2017;143:1263-73.

22. Fearon K, Arends J, Baracos V. Understanding the mechanisms and treatment options in cancer cachexia. Nat Rev Clin Oncol 2013;10:90-9.

23. Shimizu K, Okita R, Saisho S, et al. Preoperative neutrophil/lymphocyte ratio and prognostic nutritional index predict survival in patients with non-small cell lung cancer. World J Surg Oncol 2015;13:291.

24. Jin S, Cao S, Xu S, et al. Clinical impact of pretreatment prognostic nutritional index (PNI) in small cell lung cancer patients treated with platinum-based chemotherapy. Clin Respir J 2018;12:2433-40.

25. Dai Y, Fu X, Li T, et al. Long-term impact of prognostic nutritional index in cervical esophageal squamous cell carcinoma patients undergoing definitive radiotherapy. Ann Transl Med 2019;7:175.

26. Gupta D, Lis CG. Pretreatment serum albumin as a predictor of cancer survival: a systematic review of the epidemiological literature. Nutr J 2010;9:69.

27. Gu XB, Tian T, Tian XJ, et al. Prognostic significance of neutrophil-to-lymphocyte ratio in non-small cell lung cancer: a meta-analysis. Sci Rep 2015;5:12493.

28. Geng Y, Shao Y, Zhu D, et al. Systemic ImmuneInflammation Index Predicts Prognosis of Patients with Esophageal Squamous Cell Carcinoma: A Propensity Score-matched Analysis. Sci Rep 2016;6:39482.

29. Keizman D, Gottfried M, Ish-Shalom M, et al. Pretreatment neutrophil-to-lymphocyte ratio in metastatic castration-resistant prostate cancer patients treated with ketoconazole: association with outcome and predictive nomogram. Oncologist 2012;17:1508-14.

30. Diakos CI, Charles KA, McMillan DC, et al. Cancerrelated inflammation and treatment effectiveness. Lancet Oncol 2014;15:e493-503.

31. Roxburgh CS, McMillan DC. Cancer and systemic inflammation: treat the tumour and treat the host. Br J Cancer 2014;110:1409-12.

32. Khunger M, Patil PD, Khunger A, et al. Post-treatment changes in hematological parameters predict response to nivolumab monotherapy in non-small cell lung cancer patients. PLoS One 2018;13:e0197743.

33. Zhang Y, Feng YC, Zhu HG, et al. The peripheral blood neutrophil-to-lymphocyte ratio is a prognostic predictor for survival of EGFR-mutant nonsmall cell lung cancer patients treated with EGFR-TKIs. Medicine (Baltimore) 2018;97:e11648.

34. Cao D, Xu H, Xu X, et al. A reliable and feasible way to predict the benefits of Nivolumab in patients with nonsmall cell lung cancer: a pooled analysis of 14 retrospective studies. Oncoimmunology 2018;7:e1507262.

Cite this article as: Wang J, Liu Y, Mi X, Shao M, Liu L. The prognostic value of prognostic nutritional index (PNI) and neutrophil to lymphocyte ratio (NLR) for advanced non-small cell lung cancer treated with platinum-based chemotherapeutics. Ann Palliat Med 2020;9(3):967-978. doi: 10.21037/apm.2020.04.31 